Reprod. Nutr. Dévelop., 1980, 20 (4 B), 1143-1148.

\title{
Effets d'un repas protéique liquide sur le débit acide et l'évacua- tion gastriques, la gastrine sérique avant et après vagotomie fundique chez l'ulcéreux duodénal
}

\author{
par Monique SAUVAGE, Ph. ROUGIER, M. MIGNON, S. BONFILS \\ Service de Gastroentérologie ef INSERM U 10 , \\ Hôpital Bichaf 170 boulevard Ney, 75877 Paris Cedex 18.
}

Summary. Action of a liquid protein meal upon acid secretion, gastrin release and gastric emptying in duodenal ulcer patients before and after proximal vagotomy.

Using a technique adapted from the intragastric titration method of Fordtran and Walsh, food-stimulated acid secretion, gastrin release and gastric emptying have been investigated before and after proximal vagotomy in 11 duodenal ulcer patients.

Vagal denervation of the proximal stomach resulted in (i) a 40 p. 100 decrease in acid secretion in response to a protein meal ; this response was of the same magnitude as the reduction in pentagastrin-stimulated acid output, (ii) a marked increase in food-induced gastrin release, suggesting a relationship between antral gastrin release and fundal vagal innervation since antral $\mathrm{pH}$ remained constant and the amount of protein and the distension volume within the antrum were the same as before proximal vagotomy, (iii) a slowing down of the initial phase of gastric emptying of the liquid protein meal in 5 patients, a slight acceleration in 4 and no change in 2 .

The clinical relevance of these findings is discussed.

\section{Introduction.}

Le «Dumping » syndrome par évacuation trop rapide du contenu gastrique est une complication fréquente de tous les types de vagotomie pour ulcère duodénal (Jordan, 1976). Cette séquelle est liée tant au procédé de drainage éventuellement associé à la vagotomie, qu'à la dénervation de l'estomac proximal dont la capacité de relaxation et d'adaptation aux volumes intragastriques est altérée et la fonction acido-secrétrice réduite. Seule la dénervation fundique paraît en jeu lors de la vagotomie supra-sélective (VSS) puisque cet acte chirurgical ne comporte pas de procédé de drainage. Les quelques études consacrées au retentissement de la VSS sur l'évacuation gastrique des liquides ont donné lieu à des résultats contradictoires (Clarke ef Alexander-Williams, 1973 ; Berger et al., 1976).

Grâce à une méthodologie permettant l'étude simultanée de la secrétion et de la vidange gastrique (Rougier, Sauvage et Mignon, 1978) nous avons cherché à apprécier 
comment la dénervation proximale de l'estomac modifiait les effets d'un repas protéique liquide sur ces paramètres, ainsi que sur la réponse gastrinique susceptible elle aussi de jouer un rôle dans l'évacuation gastrique (Cohen, Long ef Snape, 1979).

\section{Sujets étudiés et méthodologie.}

Onze sujets (9 hommes, 2 femmes) d'âge moyen 40 ans (extrêmes 20-72), porteurs d'un ulcère duodénal évolutif au moment de l'étude, authentifié endoscopiquement, ont été l'objet de mesures de la secrétion acide et de l'évacuation gastrique des liquides avant, ef 3 mois après avoir subi une vagotomie supra-sélective.

1. Etude secrétoire. - Chaque sujet a été étudié à deux reprises selon 2 protocoles : - la titration intragastrique et - le fubage gastrique conventionnel. Dans les 2 cas, le sujet est placé en décubitus latéral gauche. Le recueil de la secrétion se fait à l'aide d'une sonde gastrique dont la bonne position est vérifiée.

La technique de titration intragastrique (Fordtran et Walsh, 1973) permet la mesure de la secrétion acide induite par l'introduction dans l'estomac d'un repas protéique liquide. Ce repas est composé de $18 \mathrm{~g}$ d'extraits protéiques Liebig $(10 \mathrm{~g}$ de protéines, $3 \mathrm{~g}$ de glucides, $0,05 \mathrm{~g}$ de lipides, 330 mOsm en solution dans $400 \mathrm{ml}$ d'eau dont le $\mathrm{pH}$ est ajusté à 4,5 à l'aide d'une solution normale d'HCl).

La sonde utilisée est une sonde en polyvinyl (16 French) sur laquelle sont accolés 2 cathéters de $2 \mathrm{~mm}$ de diamètre interne. Après vidange de l'estomac pendant 15 min ef recueil de la secrétion basale pendant $60 \mathrm{~min}$, le repas est introduit par la sonde en l'espace de $3 \mathrm{~min}$. L'homogénéité du contenu gastrique est assurée par aspirationréinjection rapides, 2 à 3 fois $/ \mathrm{min}$. Le $\mathrm{pH}$ intragastrique est mesuré toutes les $2 \mathrm{~min}$. Pour maintenir le $\mathrm{pH}$ constant à $4,5, \mathrm{HCO}_{3}-\mathrm{Na} 0,4 \mathrm{~N}$ est instillé à débit variable par l'un des cathéters. La quantité de carbonate monosodique ajoutée correspond à la quantité de mmole d' $\mathrm{H}^{+}$secrété. Le fest conventionnel à la Pentagastrine $(6 \mu \mathrm{g} / \mathrm{kg} / \mathrm{M})$ est réalisé selon les conditions habituelles de notre laboratoire (Mignon, Leroux ef Rougier, 1978). Il sert de référence pour juger de la qualité de la vagotomie. Les résultats sont exprimés en terme de débit acide basal $60 \mathrm{~min}$ (DAB $60 \mathrm{~min}$, en mEq), de pic acide horaire ( $\mathrm{PAH}$ en $\mathrm{mEq}$ ) pour la réponse à la Pentagastrine et de débit acide horaire en réponse au repas protéique (DAH TIG, en $\mathrm{mEq}$ ) correspondant à la secrétion mesurée pendant la 1 re heure du test.

2. Efude de l'évacuation gastrique des liquides. - Elle consiste à suivre l'évolution de la quantité de repas restant à l'intérieur de l'estomac au cours de la TIG par des mesures à 10, 25, 45, 75 min : a) du volume intragastrique (VIG) ; b) de la concentration en protéines du contenu gastrique. Le produit du VIG $X$ la concentration protéique permet le calcul du volume de repas restant (VRR) dans l'estomac. La mesure du VIG utilise la technique de dilution d'un indicateur coloré, le rouge de phénol, selon la formule $V I G=\frac{V_{2}\left(C_{2}-C_{3}\right)}{C_{3}-C_{1}}$ où $V_{2}$ et $C_{2}$ sont respectivement le volume et la concentration de la solution de rouge de phénol ajoutée, $C_{1}$ la concentration de rouge de phénol avant addition de $V_{2} C_{2}$ et mélange énergique du contenu gastrique à la seringue. La concentration de rouge de phénol est mesurée au spectro-photomètre à 
$560 \mathrm{~nm}$. La concentration en protéines est évaluée au spectro-photomètre à $278 \mathrm{~nm}$; les mesures sont effectuées à partir d'aliquots $(2 \mathrm{ml})$ de liquide gastrique prélevés immédiatement avant les mesures du VIG. L'absorption à $278 \mathrm{~nm}$ du rouge de phénol est faible et systématiquement soustraite des mesures précédentes à partir d'abaques. La quantité de repas restant dans l'estomac aux différents temps de l'étude est exprimée en p. 100 de la quantité de repas introduite. Le paramètre retenu pour la mesure de l'évacuation initiale du repas liquide est le « $t$ » $1 / 2$ qui correspond au temps de demiévacuation du repas liquide lu sur la courbe cinétique obtenue.

3. Mesure de la réponse gastrinique. - La gastrine immuno-réactive est mesurée en basale au temps : $-30,-15$ et 0 et durant le titration à $5,10,15,30,60$ min après l'ingestion du repas. Les variations gastriniques en réponse au repas protéique sont exprimées en terme de réponse gastrinique intégrée (RIG) exprimée en $\mathrm{pg} / \mathrm{ml} / 60 \mathrm{~min}$.

4. Statistique. - Les différences entre les valeurs des résultats obtenues avant et après VSS ont faif l'objet d'une analyse de variance pour données appariées et la signification des différences, a été testée à l'aide d'un « $t$ » de Student. Le niveau de signification statistique choisi est $\mathrm{p}<0,05$.

\section{Résultats.}

1. Réponse secrétoire acide. - Le tableau 1 indique les valeurs de la réponse acide au repas protéique avant et quelques mois après VSS. Les valeurs des DAB 60 min et des PAH pentagastrine ont été également rapportés dans ce tableau dans la mesure

\section{TABLEAU 1}

Débit acide a) basal horaire $(D A B H)$ b) en réponse au repas protéique $(D A H T / G)$ c) après $6 \mu \mathrm{g} / \mathrm{kg} / I M$ de Pentagastrine (PAH Penta) avant ef 3 mois après VSS chez 11 molades porteurs d'un ulcère duodénal

\begin{tabular}{|c|c|c|c|c|c|c|}
\hline \multirow{2}{*}{ 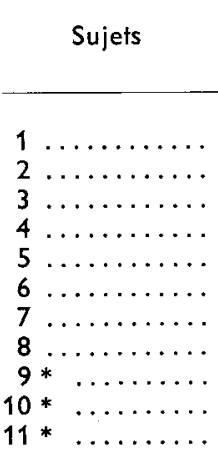 } & \multicolumn{2}{|c|}{$\begin{array}{c}\text { DABH mEq } \\
\text { pré VSS post VSS }\end{array}$} & \multicolumn{2}{|c|}{$\begin{array}{l}\text { DAH TIG mEq } \\
\text { pré VSS post VSS }\end{array}$} & \multicolumn{2}{|c|}{$\begin{array}{l}\text { PAH Penta mEq } \\
\text { pré VSS post VSS }\end{array}$} \\
\hline & $\begin{array}{c}0 \\
17 \\
6,8 \\
11,8 \\
2,2 \\
7,5 \\
3,6 \\
0 \\
0,9 \\
9 \\
11\end{array}$ & $\begin{array}{l}0 \\
4,1 \\
3 \\
2 \\
0 \\
2,8 \\
0 \\
0 \\
2,5 \\
0,4 \\
2\end{array}$ & $\begin{array}{l}30,8 \\
45 \\
33,4 \\
39,6 \\
36,4 \\
34 \\
43,6 \\
17,9 \\
23,4 \\
34 \\
31,2\end{array}$ & $\begin{array}{c}8,7 \\
30,8 \\
30,2 \\
27,8 \\
21,1 \\
28,8 \\
24 \\
4,8 \\
13,6 \\
16,8 \\
17,4\end{array}$ & $\begin{array}{l}22,5 \\
54,4 \\
35,5 \\
19,1 \\
32 \\
32,9 \\
26 \\
29,2 \\
- \\
-\end{array}$ & $\begin{array}{c}8,4 \\
28,3 \\
30,2 \\
9,8 \\
20 \\
27,3 \\
20 \\
4,1 \\
- \\
-\end{array}$ \\
\hline $\begin{array}{l}\mathrm{m} \ldots \ldots \ldots \ldots \\
\pm \sigma \quad \ldots \ldots \ldots\end{array}$ & $\begin{array}{r}6,3 \\
+5,5\end{array}$ & $\begin{array}{r}1,5 \\
+\quad 1,5\end{array}$ & $\begin{array}{r}33,6 \\
\pm \quad 8,0\end{array}$ & $\begin{array}{r}20,4 \\
\pm \quad 8,9\end{array}$ & $\begin{array}{r}31,4 \\
+10,7\end{array}$ & $\begin{array}{l}18,5 \\
\pm 10\end{array}$ \\
\hline
\end{tabular}

\footnotetext{
* Ont refusé le test-Pentagastrine.
} 
où ils permettent d'apprécier la qualité de la dénervation fundique. La réduction moyennie de la réponse acide au repas protéique après VSS est de 41,3 $\pm 19,9$ p. 100 $(\sigma)$ analogue à celle de la réponse à la stimulation maximale exogène par la pentagastrine qui est de $41 \pm 24,3$ p. 100. La réduction moyenne apportée par la VSS au débit acide basal est de $82,1 \pm 5,6$ p. 100 après exclusion du sujet 9 où il existe une augmentation du DAB post-opératoire. Les réductions induites par la VSS aux réponses acides au repas protéique d'une part, à la pentagastrine d'autre part, sont étroitement correlées $r=0,83, p<0,01$ et il n'existe pas de différence sigrificative entre les valeurs post-opératoires des DAH TIG el des PAH pentagastrine.

2. Evacuation du repas protéique liquide. - Comme l'indique la figure 1 la cinétique moyenne de l'évacuation gastrique du repas protéique est peu altérée par la VSS. Le $+1 / 2$ moyen après VSS est légèrement allongé $35,2 \pm 26,2$ min contre $23,8 \pm 12,3$ avant VSS, mais il existe de grandes variations individuelles ai:asi que l'indiquel'encart de la figure et la différence entre les deux $11 / 2$ moyens n'est pas significative $p>0,05$. Il est à noter que dans les cas où il y a eu après VSS une accélération de l'évacuation initiale des liquides, les malades ne se sont pas plaints de «dumping » syndrome. De même un seul malade présentant un allongement important de l'évacuation initiale du repas protéique liquide présentait une sensation de lourdeur gastrique après le repas.

II n'y a pas après VSS de relation apparente entre les variations de l'évacuation gastrique et les réductions de la réponse acide au repas protéique liquide.

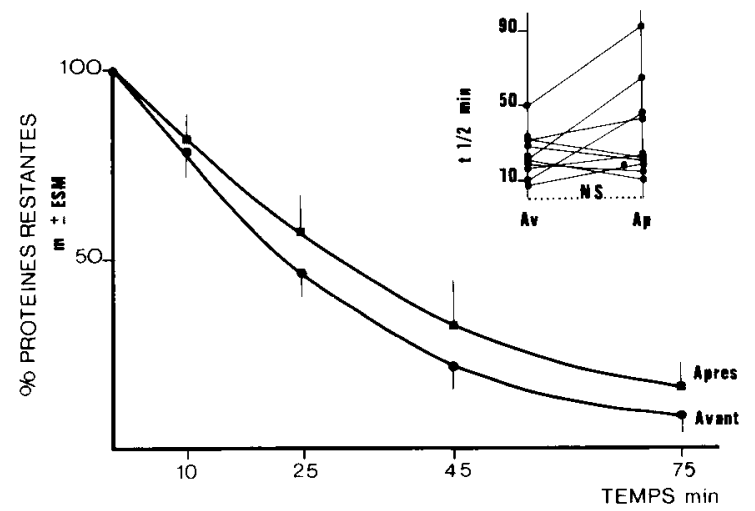

FIG. 1. - Evolution au cours de la TIG de la quantité de repas protéique restant dans l'estomac, exprimée en p. 100 de la quantité initiale chez 10 ulcéreux duodénaux avant ef après VSS. L'encart de la figure indique les temps de demi-évacuation du repas test, avant et après VSS.

$3^{\circ}$ Réponses gastriniques au repas protéique. - Chez tous les sujets la RIG est augmentée par la dénervation fundique : la valeur moyenne post-VSS est de $12991 \pm 7605 \mathrm{pg} / \mathrm{ml} / 60 \mathrm{~min}$ versus $1415 \pm 1686$ avant VSS. II n'existe pas de corrélation entre les variations post-opératoires des vitesses d'évacuation initiale du repas protéique et celles des réponses gastriniques intégrées. 


\section{Discussion.}

Le but de la VSS étant de réduire la secrétion gastrique acide dans toutes les circonstances la stimulant, en particulier l'alimentation, la réduction de la réponse acide au repas protéique engendrée par la VSS chez nos 11 sujets était prévisible. Cette réduction de la stimulation endogène de la secrétion acide gastrique est comparable, dans ce travail, à celle de la stimulation exogène de l'estomac. En ce qui concerne la réduction moyenne du PAH Pentagastrine, nos résultats sont dans les limites des résultats de la littérature (Jordan, 1976). Par contre, la réponse acide au repas protéique est moins diminuée par la VSS dans notre groupe de sujets que dans celui de 7 malades ulcéreux duodénaux étudiés par Feldman et al., 1979. II n'y a pas d'autres études équivalentes dans la littérature pour l'instant. La différence entre les 2 groupes de résultats peut être en partie due à une différence de technique opératoire : pour Feldman ef al., 1979, en effet, la dénervation du fundus s'accompagne aussi d'une énervation des 7 derniers centimètres de l'œsophage. Il est vraisemblable aussi à en juger sur les valeurs du débit acide basal et la réponse à la Pentagastrine que certains de nos sujets ont une dénervation fundique vagale incomplète. Il est nécessaire d'attendre que de nombreux malades subissent ce contrôle post-opératoire pour apprécier réellement comment la dénervation élective du fundus altère la réponse acide qu'induit l'alimentation, représentée dans ce travail par un repas protéique liquide, élément de stimulation optimale de la secrétion gastrique acide.

En ce qui concerne l'évacuation gastrique du repas protéique liquide, si nos résultats indiquent dans l'ensemble que la VSS n'entraîne pas de modifications majeures, il existe toutefois chez 3 sujets un ralentissement considérable de l'évacuation initiale et totale du repas protéique liquide bien que les caractéristiques $d u$ repas test utilisé soient, sur le plan du volume, de l'osmolarité ef de la charge calorique, celles qui permettent une vidange gastrique optimale (Hunt and Spurrell, 1951 ; McHugh et Moran, 1979). L'anomalie motrice notée chez ces malades n'apparaît, en tout cas, pas liée aux modifications post-VSS de la secrétion acide ni de la réponse gastrinique.

Dans les conditions de notre étude, l'accroissement constamment noté de la réponse gastrinique à l'introduction du repas protéique constitue un fait intéressant à discuter et difficile à expliquer. Cette augmentation ne paraît pas liée aux éléments suivants : a) stase antrale, source de distension et, par voie de conséquence, de libérafion accrue de gastrine en réponse aux protéines du repas, puisque, du fait de la réduction secrétoire acide, l'importance des volumes intragastriques esł plus faible ; b) réduction de l'efficacité du mécanisme antral physiologique de régulation gastrinique $\mathrm{pH}$-dépendant, car la technique employée dans notre travail maintient un $\mathrm{pH}$ consiant à 4,$5 ; c)$ plus grande quantité de protéines restant dans l'estomac puisqu'il n'y a pas de différence significative entre les valeurs successives des quantités de repas protéique restant dans l'estomac au cours de la TIG (fig. 1).

Deux autres mécanismes peuvent être proposés hypothétiquement pour expliquer les modifications post-VSS de la réponse gastrinique au repas protéique : a) la dénervation vagale elle-même, ce qui suppose que, par des voies à déterminer, les fibres vagales du fundus exerceraient un contrôle négatif sur le fonctionnement du mécanisme gastrinique antral. La mise en évidence de fibres vagales inhibitrices à destinée 
antrale (Farooq et Walsh, 1975) apporte un début de soutien à une telle hypothèse ; b) développement d'une hyperplasie des cellules à gastrine au niveau de l'antre ; mais il n'y a encore aucun argument sérieux en faveur d'un tel concept.

Les conséquences de ceffe hyperproduction de gastrine au repas sont encore difficiles à appréhender. L'absence de corrélation des RGI avec d'une part l'évacuation gastrique, d'autre part les réponses secrétoires (ce travail, Bonfils, Mignon, Rozé, 1979), semble réduire l'importance de ce phénomène dans les suites proches de la VSS ; on peut s'interroger, toutefois sur les conséquences à long terme des réponses gastriniques de grande amplitude en évoquant le rôle trophique de la gastrine, en particulier sur la masse cellulaire pariétale de l'estomac.

En conclusion : après dénervation sélective de l'estomac proximal les effets d'un repas protéique liquide sont caractérisés par a) réduction de la réponse secrétoire acide d'environ 40 p. 100 b) augmentation très nette de la réponse gastrinique faisant discuter un possible contrôle vagal du mécanisme gastrinique, de siège fundique ; c) absence de modification de l'évacuation initiale du repas test, bien que quelques sujets aient un ralentissement important de celle-ci, d) les modifications des réponses acide et gastrinique n'interviennent pas dans les variations de l'évacuation gastrique du- repas protéique liquide testé.

\begin{abstract}
Journées Ingestion-Digestion-Absorption de l'Association française de Nutrition Paris, 15-16 novembre 1979.
\end{abstract}

\title{
Références
}

BERGER T., CEDER L., HAMFELT A., MEURLING S., 1976. Effect of highly selective vagotomy on gastric emptying. Scand. J. Gastroenterol., 11, 829-832.

BONFILS S., MIGNON M., ROZÉ Cl., 1979. Vagal control of gastric secretion, 59-106. In CRANE R. K., Int. Rev. Physiol., Gastro-intestinal physiology, III, vol. 19, Univ. Park Press, Baltimore.

CLARKE R. J., ALEXANDER-WILLIAMS J., 1973. The effect of preserving antral innervation and of pyloroplasty on gastric emptying after vagotomy in man. Gut, 14, 300-307.

COHEN S., LONG W. B., SNAPE W. J., 1979. Gastrointestinal motility, 107-149. In CRANE R. K., Int. Rev. Physiol., Gastro-infestinal physiology, III, vol. 19, Univ. Park Press, Baltimore.

FAROOQ O., WALSH J. H., 1975. Atropine enhances serum gastrin response to insulin in man. Gastroenterology, 68, 662-666.

FELDMAN M., DICKERMAN R. M., MCCLELLAND R. N., COOPER K. A., WALSH J. H., RICHARDSON C. T., 1979. Effect of selective proximal vagotomy on food stimulated gastric acid secretion and gastrin release in patients with duodenal ulcer. Gastroenterology, 76, 926-931.

FORDTRAN J. S., WALSH J. H., 1973. Gastric acid secretion rate and buffer content of the stomach after eating. Results in normal subjects and in patients with duodenal ulcer. J. clin. Invest., 52, 645-657.

HUNT J. N., SPURRELL W. R., 1951. The pattern of emptying of the human stomach. J. Physiol. (London), 113, 157-168.

JORDAN P. H., 1976. Current status of parietal cell vagotomy. Ann. Surg., 184, 659-671.

McHUGH P. R., MORAN T. H., 1979. Calories and gastric emptying : a regulatory capacity with implications for feeding. Am. J. Physiol., 236, R 254-R 260.

MIGNON M., LEROUX S., ROUGIER P., 1978. Indications et résultats de l'exploration de la secrétion gastrique acide par tubage chez l'homme adulte, 44-73. In GIRARD M. L., ROUSSELET F., Biologie de la digestion. Explorations fonctionnelles. APDILA Paris.

ROUGIER P., SAUVAGE M., MIGNON M., 1978. Titration intra-gastrique : méthode d'étude simultanée de la secrétion ef de la vidange gastrique, premiers résultats, 74-85. In GIRARD M. L., ROUSSELET F., Biologie de lo digestion. Explorations fonctionnelles. APDILA Paris. 